\title{
Abstracts from the ASENT 2008 Annual Meeting March 6-8, 2008
}

\author{
Poster Abstract \#1: Clinically Meaningful \\ Differences of the Ashworth Scale in Post-Stroke \\ Upper Limb Spasticity \\ Amanda VanDenburgh*, Manasee V. Shah*, S. Hua*, \\ S. Abu-Shakra*, Jon Wagg ${ }^{\dagger}$, Tatiana Khariton ${ }^{\dagger \ddagger}$, \\ F.C. Beddingfield III** ${ }^{*}$, and M.F. Brin*\$ \\ *Global Clinical Development, Allergan, Irvine, CA; \\ ${ }^{\dagger}$ Pharsight, Mountain View, CA; ${ }^{\ddagger}$ Department of Medicine, \\ David Geffen School of Medicine at University of California \\ Los Angeles, Los Angeles, CA; ${ }^{\S}$ Department of Neurology, \\ University of California Irvine, Irvine, $C A$
}

Background and Objective: An assessment of clinically important change is useful in interpreting clinical trial outcomes. The 4-point Ashworth Scale (AS) is a widely accepted measure of muscle tone, and the 9-point Physician Global Assessment Score (PGAS) is a global measure of post-treatment change as evaluated by the physician. The quantitative relationship between AS and PGAS has not previously been described in spasticity.

Methods: Data from three clinical trials of botulinum toxin type A (BOTOX; Allergan, Irvine, CA) in poststroke spasticity were analyzed $(n=442)$. Mean change from baseline in AS score was plotted as a function of PGAS and correlations were calculated. Receiver-operator curve (ROC) analyses with the wrist flexor AS change from baseline as the independent variable and PGAS as the dependent variable were performed using the following criteria: PGAS of $\geq 1$ (mild improvement or better) and PGAS of $\geq 2$ (moderate improvement or better).

Results: In pooled data, the Pearson's correlation coefficient $r$ between the change in wrist Ashworth Score and the PGAS was $-0.44(p=0, t=-26.5)$. Pearson correlations by individual study were also statistically significant. By ROC analysis, a PGAS of $\geq 1$ was associated with $33 \%$ reduction of wrist AS and a PGAS of $\geq 2$ was associated with approximately $50 \%$ reduction. In a well-powered phase 3 study, the Pearson's correlation coefficient was even higher $(r=-0.76, p=0, t=-28.5)$.

Conclusions: Changes in disease-specific scales that correlate significantly with changes in physician global assessments are considered clinically meaningful. In clinical trials of chronic spasticity, we found that $33 \%$ and $50 \%$ changes in the Ashworth Scale scores correlate with 1-point or 2-point changes in PGAS. This is similar to findings from pooled chronic pain studies, in which $30 \%$ and $50 \%$ changes on the pain numeric rating scale were considered clinically important (Farrar et al., Pain 2001;94:149-158).

Study supported by Allergan, Irvine, CA.

\section{Poster Abstract \#2: Surrogate Completion of the Treatment Satisfaction Questionnaire for Medication for a Trial of Corticosteroids in Duchenne Muscular Dystrophy}

Barbara E. Herr*, Kimberly A. Hart*, Michael McDermott*, Robert C. Griggs*, Laura Herbelin ${ }^{\dagger}$, and Richard Barohn ${ }^{\dagger}$

*University of Rochester, Rochester, $N Y ;{ }^{\dagger}$ University of Kansas, Kansas City, KS
Introduction: Satisfaction with treatment has become a useful outcome measure in oncologic studies, in which severe side effects must be balanced with tumor regression to determine if the treatment is of overall benefit to a patient. The use of corticosteroids in Duchenne muscular dystrophy (DMD) poses a similar dilemma: Does the improvement in strength outweigh the inevitable side effects and need for life-long medication?

Background: We are planning an international, randomized, double-blind trial in 300 DMD boys aged 4 to 7 years to compare three corticosteroid regimens with respect to function and patient-parent satisfaction over a 3- to 5-year follow-up period. Global treatment satisfaction will be measured by the Treatment Satisfaction Questionnaire for Medication (TSQM), which is a patient-centered assessment of satisfaction with treatment that is particularly relevant to a noncurative therapy applied in a chronic disease. The hypothesis is that a patientparent satisfaction measure as a component of the primary outcome variable can yield firm evidence for changing practice and developing a standard of care. The questionnaire is designed to be completed by the individual with the chronic disease. Because the DMD boys are too young to complete the form, parents will serve as proxies, answering the questionnaire as they think their sons would.

Pilot Study: The TSQM has been validated only in adults with chronic disease, and only with the patient as responder. We asked 50 parents to complete the questionnaire from the perspective of their DMD sons taking corticosteroids. Parents also provided the son's age, walking ability, medications, and a rating of ease in completing the TSQM from the son's perspective.

Results: The results show that the parents felt that they could easily report on behalf of the sons. Associations between TSQM responses and demographic information (e.g., age, ambulatory status) will be analyzed for a future report.

Sponsored in part by the National Institute of Neurological Disorders and Stroke, National Institutes of Health.

\section{Poster Abstract \#3: mGluR5 Activation Reduces Microglial-Mediated Neurotoxicity in Vitro and Improves Outcome After Experimental Traumatic Brain Injury}

David J. Loane, Kimberly R. Byrnes, Bogdan A. Stoica, Ahdeah Pajoohesh-Ganji, and Alan I. Faden

Department of Neuroscience, Georgetown University Medical Center

In traumatic brain injury, neuronal cell death continues long after the initial insult; persistent activation of microglial cells may contribute to this process. Metabotropic glutamate receptors (mGluRs) are G-protein coupled receptors. Group I mGluRs modulate neuronal cell death: mGluR1 activation enhances necrotic cell death and mGluR5 activation inhibits apoptosis. We have found that microglia strongly express mGluR 5 but not mGluR1, and speculated that they may serve to modulate microglial-mediated inflammation and neuronal toxicity. We explored this question using both primary rat 
microglial cultures and a mouse microglial cell line (BV2). Stimulation of BV2 microglia by lipopolysaccharide (LPS) increased inducible nitric oxide synthase expression and nitric oxide production, a key inflammatory signaling molecule. Pretreatment with the mGluR5 agonist CHPG significantly attenuated these responses, which were reversed by the mGluR5 antagonist MTEP. LPS stimulation also increased the expression of p22PHOX, a component of the NADPH oxidase enzyme complex that generates reactive oxygen species; both p22PHOX and reactive oxygen species were attenuated by CHPG. Conditioned media from LPS-stimulated BV2 microglia significantly increased neuronal cell death, which was blocked by CHPG pretreatment. Similar observations were made using primary microglial cultures. After controlled cortical impact injury, mice received intracerebroventricular injection of either CHPG or vehicle at 30 minutes after injury. CHPG significantly reduced lesion volume (by T2-weighted magnetic resonance imaging) at 3 weeks and improved motor function and spatial learning performance when compared to vehicle-treated mice; each of the effects was blocked by MTEP. Moreover, at 21 days post injury, the CHPG-treated mice showed a marked reduction in ED1 expression, a marker of activated microglia. These data support a potential therapeutic role of mGluR5 agonists for inhibiting microglial activation and related neurotoxicity. Given the important proposed role of neuroinflammation in acute and chronic neurodegeneration, these observations may have broader implications for many neurological disorders.

\section{Poster Abstract \#4: Development of Long-Duration Accelerometry for Use in Clinical Trials of Essential Tremor}

Fatta Nahab, Loretta Wittevrongel, and Mark Hallett

National Institute of Neurological Disorders and Stroke, National Institutes of Health, Bethesda, MD

Background: Essential tremor (ET) is a common movement disorder affecting $0.4 \%$ of the general population and $14 \%$ of people 65 years and older. Current therapies provide modest tremor suppression and are often accompanied by intolerable side effects. Key to the success of future studies is the development of objective biomarkers to assess tremor in a sensitive and unbiased manner. Numerous subjective and objective tremor measures are available, including laboratorybased accelerometry, electromyography, spirography, and surveys assessing quality of life or impact on activities of daily living. The utility of objective measures are limited by time and expense, and subjective measures lack sensitivity and rely heavily on standardization of ratings or patient responses. Furthermore, all these methods are temporally biased, in that they reflect only brief time intervals. We hypothesized that portable uniaxial monitoring devices could be used to reliably measure tremor over long durations in patients with ET.

Methods: The study population consisted of adults with ET who underwent an ethanol challenge to assess tremor response, prior to enrollment in a clinical trial at NIH. Wristbased accelerometers were used (Cambridge Neurotechnology, Cambridge, UK) to record the intensity and duration of tremor and nontremor movements. We developed an algorithm to calculate a Tremor Severity Score (TSS) characterizing tremor patterns over extended time periods. To assess individual subject changes, we normalized the TSS at each timepoint by each subject's own baseline TSS. The primary outcome measures were TSS at baseline, $0-30,30-60$, and 60-90 minutes after ethanol challenge.
Results: Thirteen patients were included in the analysis. Mean percent reduction in tremors from baseline TSS was $165 \%, 408 \%$, and $423 \%$ at $0-30,30-60$, and $60-90$ minutes after ethanol challenge, respectively. A post hoc linear regression of the TSS total score and baseline TSS revealed high correlation $\left(R^{2}=0.77\right)$.

Conclusions: Our results show that the TSS, based on long-duration accelerometry data, provides a sensitive and reliable method for objectively measuring ET outcomes. This method could serve as a model for the development of lowcost, high-throughput clinical trials. Further work is needed to validate this method with tremor measures in current use.

\section{Poster Abstract \#5: NIH Roadmap Molecular Libraries Screening Centers Network}

\author{
Ingrid $\mathrm{Li}^{*}$, Linda Brady*, Jamie Driscoll*, and \\ Mark Guyer ${ }^{\dagger}$; Yong Yao \\ $*$ National Institute of Mental Health and ${ }^{\dagger}$ National Human \\ Genome Research Institute, National Institutes of Health, \\ Bethesda, MD
}

Background: The NIH Roadmap is designed to enable the rapid transformation of new scientific knowledge into tangible benefits for public health. The Molecular Libraries Initiative is one of the initial projects developed under the New Pathways to Discovery roadmap theme. The Molecular Libraries Screening Centers Network (MLSCN) offers the research community an opportunity to leverage biology and chemistry resources to identify small molecule probes for innovative or challenging targets with the potential for insight into biological pathways that affect public health.

Methods: The MLSCN performs high-throughput screening (HTS) of biochemical or cell-based assays solicited from the scientific community against a high-quality library of $>150,000$ compounds maintained in a shared repository, which is expanding up to 500,000 with unique compounds based on known biological targets, active ingredients of FDA-approved drugs, clinical candidates, chemically diverse compounds with SAR clusters of 3-5, and natural product scaffolds (all with $>90 \%$ purity, $>10 \mathrm{mg}, \pm$ rule of 5 , solubility $>20 \mu \mathrm{g} / \mathrm{mL}$, and most importantly, QCed). The network provides synthetic chemistry capabilities to optimize hits identified in the initial screening to produce chemical probes that can be used for in vitro studies to interrogate the targets or phenotypes studied in the assays.

Results: Over the past 2 years, the MLSCN has implemented a diverse array of HTS assays, developed the capacity to conduct nearly 100 assay campaigns per year against the library of 150,000 compounds, and generated more than 13 million data points in PubChem. The HTS screening data from the MLSCN assays, including functional and pharmacologic selectivity data, are deposited into an open access database, PubChem (http:// pubchem.ncbi.nlm.nih.gov). The assays screened to date span a variety of target classes (e.g., GPCRs, ion channels, transporters, enzymes, kinases) and methods (protein-protein interactions, cell phenotypes, and model organisms) in understudied areas of disease. Chemical probes have been developed for a number of targets, including 1) voltage-dependent potassium channel beta subunit (Kvb) (SID 856002, commonly know as ebselen, $\mathrm{EC}_{50}=$ $25.8 \mu \mathrm{mol} / \mathrm{L}$ and cell permeable); 2) glucocerebrosidase (GC), $\left(\mathrm{CID} 4264637, \mathrm{IC}_{50}=75 \mathrm{nmol} / \mathrm{L}\right)$; 3) muscarinic receptor subtypes; 4) new estrogen receptor GPR30; and 5) sphingosine-1phosphate receptor subtypes. Over time, the use of the shared library by the MLSCN should provide extensive biological anno- 
tation, generating a unique and rich dataset available in the public domain.

Conclusions: The MLSCN, along with PubChem and the MLSMR, offers a new dimension in research opportunities for pharmacologists, chemists and biologists in the academic and nonprofit sector. The sharing of small molecules, biological assays, and screening data with the larger scientific community represents a new public sector paradigm that promises to facilitate the understanding of basic biological mechanisms and shorten the timeline for drug development, with resulting benefits to public health, especially for rare and neglected disorders.

\section{Poster Abstract \#6: Absorption, Distribution, Metabolism, and Excretion of ${ }^{14} \mathrm{C}$-labeled Nebicapone (BIA 3-202) and Metabolites}

Luis Almeida, Joana Maia, and Patricio Soares-da-Silva BIAL, S. Mamede do Coronado, Portugal

Background and Objective: Nebicapone [BIA 3-202; 1-(3,4-dihydroxy-5-nitrophenyl)-2-phenyl-ethanone] is a reversible catechol- $O$-methyltransferase (COMT) inhibitor developed to extend duration of action of levodopa in therapy of Parkinson's disease. The objective of this study was to investigate absorption, distribution, metabolism, and excretion of $\left[{ }^{14} \mathrm{C}\right]$ nebicapone and metabolites.

Methods: In this open-label study, a single dose of 2.29 $\mathrm{MBq}{ }^{14} \mathrm{C}$-labeled nebicapone $(200 \mathrm{mg}$ ) was orally administered to four healthy male volunteers.

Results: Metabolites were identified in plasma (8), urine (22), and feces (4), with BIA 3-476 (glucuronide) as the ubiquitous major metabolite. The plasma concentration-time profile of $\left[{ }^{14} \mathrm{C}\right]$ nebicapone demonstrated $t_{\max } 1.25 \pm 0.65 \mathrm{~h}, t_{1 / 2} 134.55 \pm$ $25.67 \mathrm{~h}, C_{\max } 19,647.02 \pm 4930.20 \mathrm{ng}-\mathrm{eq} / \mathrm{g}, \mathrm{AUC}_{0-t} 161,735.51 \pm$ 9224.66 h.ng-eq/g, and $\mathrm{AUC}_{0_{-}} 199,603.30 \pm 16,854.08 \mathrm{~h} . \mathrm{ng}-\mathrm{eq} / \mathrm{g}$. For whole blood, the values were $t_{\max } 1.00 \pm 0.41 \mathrm{~h}, t_{1 / 2} 32.98 \pm$ $22.82 \mathrm{~h}, C_{\max } 8411.21 \pm 1628.35 \mathrm{ng}-\mathrm{eq} / \mathrm{g}, \mathrm{AUC}_{0-t} 35,539.23 \pm$ $13,664.87$ h.ng-eq/g, and $\mathrm{AUC}_{0_{-}} 36,970.64 \pm 14,559.17 \mathrm{~h} . n g-e q / g$. Plasma pharmacokinetics of nebicapone demonstrated $t_{\max }$ $1.00 \pm 0.41 \mathrm{~h}, t_{1 / 2} 2.34 \pm 0.51 \mathrm{~h}, C_{\max } 12,650.00 \pm 2898.85 \mathrm{ng}-\mathrm{eq} / \mathrm{g}$, $\mathrm{AUC}_{0-t}$ 18,719.96 \pm 734.18 h.ng-eq/g, $\mathrm{AUC}_{0-} 18,392.12 \pm 753.81$ h.ng-eq/g. BIA 3-476 demonstrated $t_{\max } 1.25 \pm 0.50 \mathrm{~h}, t_{1 / 2} 3.47 \pm$ $0.68 \mathrm{~h}, C_{\max } 15,250 \pm 2563.20 \mathrm{ng}$-eq/g, $\mathrm{AUC}_{0-t} 53,810.61 \pm$ 7358.81 h.ng-eq/g, $\mathrm{AUC}_{0_{-}} 54,541.21 \pm 7135.70$ h.ng-eq/g. BIA 3-270 (methylated metabolite) demonstrated $t_{\max } 21.01 \pm 6.01 \mathrm{~h}$, $t_{1 / 2} 103.43 \pm 6.01 \mathrm{~h}, C_{\max } 286.25 \pm 20.48 \mathrm{ng}-\mathrm{eq} / \mathrm{g}, \mathrm{AUC}_{\mathrm{O}-t}$ 27,641.89 \pm 4569.99 h.ng-eq/g, $\mathrm{AUC}_{0-} 36,968.12 \pm 4294.42$ h.ng-eq/g. Thus nebicapone and BIA 3-476 account for most of the early phase circulating nebicapone-derived moieties, have limited circulating cell association, peak concentrations shortly after dosing, and short body residence. Longer terminal half-life phases were dominated by low concentrations of BIA 3-270. Urinary excretion, mainly as BIA 3-476, accounted for approximately $79 \%$ of recovered radioactivity or $70.1 \%$ of the administered dose, with excretion of unchanged nebicapone insignificant $(<1 \%)$. Fecal excretion accounted for approximately $21.4 \%$ of recovered radioactivity or $19 \%$ of the administered dose. Recovery of nebicapone associated radioactivity was $87.1 \%$, suggesting no worrisome retention. The treatment was very well tolerated, with no reported adverse events.

Conclusions: The primary metabolic pathway of nebicapone is glucuronidation. The kidney is the principal route of excretion of nebicapone metabolites.

Supported by BIAL-Portela \& C a $^{\mathrm{S} A}$.
Poster Abstract \#7: PharmacokineticPharmacodynamic Interaction Between Nebicapone (BIA 3-202) and Controlled-release Levodopa/Benserazide (Madopar HBS 125)

Luis Almeida*, Jose Francisco Rocha*, Carlos Lopes*, Teresa Nunes*, Rita Machado*, Raquel Costa*, Leonel Torrão*, Ana-Isabel Loureiro*, Manuel Vaz-da-Silva*, Lyndon Wright*, Patricio Soares-da-Silva*, and Amilcar Falcão ${ }^{\dagger}$

*BIAL, S. Mamede do Coronado, Portugal; ${ }^{\dagger} 4$ Health Limited, Cantanhede, Portugal

Background and Objective: Nebicapone (formerly known as BIA 3-202) is a new catechol- $O$-methyltransferase (COMT) inhibitor in development as a levodopa-sparing agent to be used in levodopa-treated Parkinson disease patients. The objective of this study was to investigate the effect of single oral doses of nebicapone $(50 \mathrm{mg}, 100 \mathrm{mg}$ and $200 \mathrm{mg}$ ) on the levodopa and 3-O-methyldopa (3-OMD) systemic exposure and erythrocyte soluble COMT (S-COMT) activity when coadministered with a single-dose of controlled-release levodopa/ benserazide 100/25 mg (Madopar HBS 125).

Methods: Double-blind, randomized, placebo-controlled, four-way crossover study in 16 healthy subjects, with a washout of 5 or more days between periods.

Results: Taking placebo as reference, levodopa $\mathrm{AUC}_{\mathrm{O}-t}$ geometric mean ratios (GMR) and 90\% confidence intervals $(90 \% \mathrm{CI})$ were $1.14(0.83 ; 1.65), 1.37(0.91 ; 1.80)$, and 1.42 $(0.97 ; 1.92)$ for nebicapone $50 \mathrm{mg}, 100 \mathrm{mg}$ and $200 \mathrm{mg}$, respectively. Regarding 3-OMD, $\mathrm{AUC}_{0-t}$ GMR and 90\%CI were 0.67 $(0.53 ; 0.80), 0.63(0.51 ; 0.77)$, and $0.55(0.49 ; 0.75)$, respectively. Maximum S-COMT inhibition occurred at $1.5 \mathrm{~h}$ after the dose and ranged from $57 \%$ with nebicapone $50 \mathrm{mg}$ to $74 \%$ with nebicapone $200 \mathrm{mg}$. There was an inverse correlation between plasma concentrations of nebicapone and S-COMT activity, and nebicapone $t_{\max }$ correlated well with $t_{\text {Emax }}$ of S-COMT inhibition. Treatments were well tolerated.

Conclusions: Single doses of $50 \mathrm{mg}, 100 \mathrm{mg}$, and $200 \mathrm{mg}$ of the novel COMT inhibitor nebicapone dose-dependently and significantly inhibited S-COMT activity, increased the bioavailability of levodopa, and reduced the formation of 3-OMD, when administered concomitantly with controlled-release levodopa/benserazide 100/25 mg (Madopar HBS 125).

Supported by BIAL-Portela \& $\mathrm{C}^{\mathrm{a}} \mathrm{SA}$.

\section{Poster Abstract \#8: Effect of Nebicapone (BIA 3-202) on the Pharmacokinetics and Pharmacodynamics of Warfarin in Healthy Subjects}

Luis Almeida*, Teresa Nunes*, Manuel Vaz-da-Silva*, Patricio Soares-da-Silva*, and Amilcar Falcão ${ }^{\dagger}$

*BIAL, S. Mamede do Coronado, Portugal; ${ }^{\dagger}$ Health Limited, Cantanhede, Portugal

Background and Objective: Nebicapone (BIA 3-202) is a new catechol- $O$-methyltransferase (COMT) inhibitor. Warfarin is a racemic mixture of the $R$ - and $S$-enantiomers, with most of the anticoagulant activity attributable to $S$ warfarin. In vitro, nebicapone showed an inhibitory effect upon CYP2C9, which is known to be responsible for the metabolism of $S$-warfarin. The objective of this study was to investigate the effect of nebicapone on warfarin pharmacokinetics and pharmacodynamics. 
Methods: In a single-center, open-label, randomized, two-way crossover study in 16 healthy volunteers, two treatment periods were separated by a washout period of 14 days. In one period, subjects received nebicapone $200 \mathrm{mg}$ thrice daily for 9 days, and a warfarin $25 \mathrm{mg}$ single dose concomitantly with the nebicapone morning dose on day 4 (Test). In the other period, a warfarin $25 \mathrm{mg}$ single dose was administered alone (Reference).

Results: Regarding $S$-warfarin, mean $C_{\max }$ and $\mathrm{AUC}_{0-t}$ were respectively $1644 \mathrm{ng} / \mathrm{mL}$ and $66,627 \mathrm{ng} . \mathrm{h} / \mathrm{mL}$ after Test, and $1739 \mathrm{ng} / \mathrm{mL}$ and 70,178 ng.h/mL after Reference. The $S$-warfarin Test/Reference geometric mean ratio (GMR) and $90 \%$ confidence interval $(90 \% \mathrm{CI})$ was $0.932(0.845$; $1.028)$ for $C_{\max }$ and $0.914(0.875 ; 0.954)$ for $\mathrm{AUC}_{0-t}$. $\mathrm{Re}-$ garding $R$-warfarin, mean $C_{\max }$ and $\mathrm{AUC}_{0-t}$ were respectively $1619 \mathrm{ng} / \mathrm{mL}$ and 92,796 $\mathrm{ng} . \mathrm{h} / \mathrm{mL}$ after Test, and 1649 ng/mL and 73,597 ng.h/mL after Reference. The $R$-warfarin Test/Reference GMR and 90\%CI was $0.973(0.878 ; 1.077)$ for $C_{\max }$ and $1.247(1.170 ; 1.327)$ for $\mathrm{AUC}_{0-t}$. No differences were found regarding the pharmacodynamic parameter INR.

Conclusions: Nebicapone showed no significant effect on the $S$-warfarin pharmacokinetics or on the coagulation endpoint (INR). A mild inhibition of the $R$-warfarin metabolism was found, but although the anticoagulant effect of $R$-warfarin is five times less potent than that of $S$-warfarin, the reported effect on $R$-warfarin metabolism is unlikely to be of clinical relevance.

Supported by BIAL-Portela \& $\mathrm{C}^{\mathrm{a}} \mathrm{SA}$.

\section{Poster Abstract \#9: Influence of the Personality Trait Anxiety on Adverse Non-Drug-Related Symptoms}

Luis Almeida*†, and Manuel Vaz-da-Silva*†, Teresa Nunes*, Amilcar Falcão ${ }^{\ddagger}$, Rui Coelho ${ }^{\S}$, and Antonio Albino-Teixeira ${ }^{\S}$

*Department of Research and Development, BIAL, S. Mamede do Coronado, Portugal; ${ }^{\dagger}$ Institute for Molecular and Cell Biology (IBMC), University of Oporto, Porto,

Portugal; ${ }^{\ddagger}$ Health Limited, Cantanhede, Portugal; ${ }^{\S}$ Service of Psychiatry and Mental Health, Faculty of Medicine, University of Oporto, Porto, Portugal

Background and Objective: Some adverse events commonly reported by participants in phase I studies are common complaints in individuals from normal populations. The objective of this study was to investigate the relation between the trait anxiety and the adverse non-drug-related complaints in a group of 117 normal subjects who matched the sociodemographic characteristics of our historical database of phase I participants.

Methods: Trait anxiety was assessed by the trait scale of the State-Trait Anxiety Inventory (STAI-T). Complaints were assessed by a questionnaire derived from the Spitzer's Patient Health Questionnaire (PHQ). Spearman's rho correlations between the STAI-T score and the complaints were performed.

Results: Significant positive linear correlations were found between the STAI-T score and the following adverse symptoms: stomach pain $(R=0.189 ; p<0.05)$; back pain $(R$ $=0.221 ; p<0.05)$; limb or joint pain $(R=0.207 ; p<0.05)$; headaches $(R=0.397 ; p<0.001)$; chest pain $(R=0.222 ; p<$ $0.05)$; dizziness $(R=0.269 ; p<0.01)$; fainting spells $(R=$ 0.365 ; $p<0.001)$; palpitations $(R=0.259$; $p<0.01)$; shortness of breath $(R=0.205 ; p<0.05)$; constipation, loose stools, or diarrhea $(R=0.213 ; p<0.05)$; nausea, gas, or indigestion $(R=0.353 ; p<0.001)$; feeling nervous or anxious $(R=0.477$; $p<0.001)$; feeling restless $(R=0.400 ; p<0.001)$; getting tired very easily $(R=0.413 ; p<0.001)$; muscle tension, aches, or soreness $(R=0.439 ; p<0.001)$; trouble falling asleep of staying asleep $(R=0.493 ; p<0.001)$; concentration difficulties $(R=0.481 ; p<0.001)$; and becoming easily annoyed or irritable $(R=0.465 ; p<0.001)$.

Conclusions: A positive correlation was found between trait anxiety and the occurrence of several adverse non-drugrelated symptoms in a group of normal healthy subjects. Those complaints are commonly reported as adverse events during participation in phase I studies. It may be then hypothesized that subjects higher in trait anxiety may be more likely to report such symptoms as adverse events during participation in phase I studies.

\section{Poster Abstract \#10: Prevalence of Adverse Non- drug-related Symptoms in Volunteers to Phase I Studies and in Normal Population}

Luis Almeida*, Amilcar Falcão ${ }^{\dagger}$, Teresa Nunes*, Rui Coelho ${ }^{\ddagger}$, Antonio Albino-Teixeira ${ }^{\ddagger}$, and Manuel Vaz-da-Silva*§

*Department of Research and Development, BIAL, S. Mamede do Coronado, Portugal; ${ }^{\dagger}$ Health Limited, Cantanhede, Portugal; ${ }^{\ddagger}$ Service of Psychiatry and Mental Health, Faculty of Medicine, University of Oporto, Porto, Portugal; ${ }^{\S}$ Institute for Molecular and Cell Biology (IBMC), University of Oporto, Porto, Portugal

Background and Objective: Some adverse non-drug-related symptoms are highly prevalent among normal populations. Therefore, such symptoms are likely to be reported as adverse events during the course of phase I studies. This study evaluated the adverse non-drug-related symptoms in a group of 192 healthy volunteers for phase I studies and a group of 117 healthy subjects who matched the volunteers group in terms of sociodemographic characteristics.

Methods: Both groups completed a questionnaire on the occurrence of complaints during the previous 4 weeks. In volunteers to phase I studies, the questionnaire was applied immediately before their admission to participation.

Results: The mostly commonly reported complaints (volunteers vs. control) were headache $(44.8 \%$ vs. $55.6 \%)$ and back pain $(41.4 \%$ vs. $52.1 \%)$. Compared with the control, subjects who volunteered for phase I studies presented significantly lower prevalence on the following symptoms: stomach pain $(p$ $=0.002)$; back pain $(p=0.008)$; limb or joint pain $(p=$ $0.003)$; headaches $(p=0.044)$; fainting spells $(p=0.005)$; palpitations ( $p=0.018)$; shortness of breath $(p=0.005)$; constipation, loose stools, or diarrhea $(p=0.033)$; nausea, gas, or indigestion $(p=0.019)$; feeling nervous or anxious $(p=$ 0.001 ); feeling restless ( $p=0.011$ ); getting tired very easily ( $p$ $<0.001)$; muscle tension, aches, or soreness $(p=0.007)$; and concentration difficulties $(p=0.003)$.

Conclusions: Participants in phase I studies are a selfselected sample with a lower tendency to report non-drugrelated adverse events than their peers from the normal population. The impact of this self-selection bias on the assessment of tolerability during phase I studies results deserves further evaluation. 


\section{Poster Abstract \#11: Brain Microdialysate and Plasma Concentrations of Inosine During Intravenous Inosine Infusion in Conscious Rats}

Noel Cusack*, Mark Hurtt*, Simon Katner ${ }^{\dagger}$, John Torchia ${ }^{\dagger}$, Bruce Solomon ${ }^{\dagger}$, Robyn Guinn ${ }^{\dagger}$, Candace Rohde ${ }^{\dagger}$, and Christine Thorne ${ }^{\dagger}$

*Alseres Pharmaceuticals, Hopkinton, MA; ${ }^{\dagger}$ BASi-Bioanalytical Systems, Inc., West Lafayette, IN

Background and Objective: Inosine is an endogenous purine nucleoside with neuroprotective and nerve regenerative properties. The literature suggests that inosine can cross the blood-brain barrier (BBB) after parental administration, but no direct measurements of CNS interstitial inosine levels have been reported. The objectives of this study were to measure the interstitial levels of inosine in the brain during intravenous infusion of inosine.

Methods: Inosine was administered in aqueous bicarbonate buffer ( $\mathrm{pH}$ 9.2) by intravenous infusion over 2 hours to two groups of male Sprague-Dawley rats ( $n=3$ per group). One group received nominal dose rates of 0 (vehicle), 100, and 300 $\mathrm{mg} / \mathrm{kg} / \mathrm{h}$, and the other received nominal dose rates of 0,500 , and $700 \mathrm{mg} / \mathrm{kg} / \mathrm{h}$. Each infusion was separated by a 2-day washout period. A microdialysis probe was inserted into the medial prefrontal cortex. Samples of CNS microdialysate and arterial blood samples were taken before the dose and at 20, 40, $60,80,100$, and 120 minutes during inosine infusion. Microdialysate and plasma inosine concentrations were measured using an LC/MS/MS assay.

Results: Plasma inosine concentrations were dose-dependently increased with maximal values up to $5.0,7.2,26,45$, and $146 \mu \mathrm{g} / \mathrm{mL}$ after inosine doses of $0,100,300,500$, and 700 $\mathrm{mg} / \mathrm{kg} / \mathrm{h}$, respectively. Basal microdialysate inosine concentrations were highly variable $(0.138-1.40 \mathrm{ng} / \mathrm{mL})$ within and between animals, and were therefore expressed as percent of the mean of two baseline values taken within 40 minutes before each infusion. No differences in microdialysate inosine percent increases were reliably seen between inosine doses of $0(90 \%$ to $175 \%), 100(113 \%$ to $146 \%)$, and 300 (96\% to $116 \%)$ $\mathrm{mg} / \mathrm{kg} / \mathrm{h}$. However, maximal increases of $526 \%$ to $632 \%$ and $574 \%$ to $653 \%$ were seen within 45 minutes after inosine doses of 500 and $700 \mathrm{mg} / \mathrm{kg} / \mathrm{h}$, respectively, and increases remained well above baseline for the remainder of these infusions.

Conclusions: Intravenous infusion of inosine at doses of 500 or $700 \mathrm{mg} / \mathrm{kg} / \mathrm{h}$ resulted in maximal inosine increases in the brain microdialysates from the rat medial prefrontal cortex of approximately $600 \%$ above predose levels. These data suggest that intravenous infusion of inosine could be a viable alternative to more invasive administrations to treat CNS disorders such as stroke, spinal cord injury, and traumatic brain injury.

Conducted by Bioanalytical Systems, Inc., for Alseres Pharmaceuticals.

\section{Poster Abstract \#12: Brain and Plasma Pharmacokinetics of Three Dopamine Transporter (DAT)-Selective Blockers After Administration of a Range of Single Oral Doses in Conscious Mice}

Noel J. Cusack*, Bruce Aungst ${ }^{\dagger}$, Lata Venkatarangan ${ }^{\dagger}$, Davette Behrens ${ }^{\dagger}$, Foster Brown ${ }^{\dagger}$, and Peter Meltzer ${ }^{\ddagger}$

*Alseres Pharmaceuticals, Hopkinton, MA; ${ }^{\dagger} Q P S$, Delaware

Technology Park, Newark, DE; ${ }^{\ddagger}$ Organix, Woburn, MA

Background and Objective: Inhibition of dopamine reuptake via the dopamine transporter (DAT) present on CNS presynaptic dopaminergic neurons is a potential therapy for the treatment of Parkinson's disease (PD). DAT blockers have previously been shown to mitigate PD-like symptoms induced by 1-methyl-4-phenyl-1,2,3,6-tetrahydropyridine (MPTP) in nonhuman primates. This study evaluated brain penetration of three DAT-selective blockers, O-620 (difluoropine), O-1369, and O-2099, in mice after administration of single oral doses.

Methods: DAT blockers were administered in aqueous phosphate buffer ( $\mathrm{pH}$ 3.0) by oral gavage at doses of 2, 5, 10, and $20 \mathrm{mg} / \mathrm{kg}$ to four groups of mice. Blood samples and whole brain specimens were taken at $0.5,1,2,4,6,8$, and 24 hours after the dose. Blood plasma and brain DAT blocker content used validated LC/MS/MS assay methodology. Pharmacokinetics analyses to determine elimination half-lives and area under the concentration $v s$. time (AUC) curves for plasma and brain were performed using WinNonlin software (Pharsight, Mountain View, CA).

Results: All three DAT blockers achieved rapid systemic and brain exposures $\left(C_{\max }\right.$ and AUC) with maximal plasma and brain concentrations obtained within 0.5 hours. Half-lives ranged from 0.3 to 2.7 hours (plasma) and 0.3 to 3.3 hours (brain). Plasma mean $C_{\max }$ values after doses of $2,5,10$, and 20 $\mathrm{mg} / \mathrm{kg}$ were $49.4,128,579$, and $1338 \mathrm{ng} / \mathrm{mL}$ (O-620); 12, 19 , 73, and $417 \mathrm{ng} / \mathrm{mL}$ (O-1369); and 172, 406, 759, and 1509 $\mathrm{ng} / \mathrm{mL}$ (O-2099). Corresponding brain mean $C_{\max }$ values were 377, 1547, 3348, and $9407 \mathrm{ng} / \mathrm{g}(\mathrm{O}-620) ; 60,131,497$, and $2584 \mathrm{ng} / \mathrm{g}(\mathrm{O}-1369)$; and 447, 1049, 2594, and $6883 \mathrm{ng} / \mathrm{g}$ (O-2099). Mean brain-to-plasma concentration ratios and mean brain-to-plasma AUC ratios ranged from 2.2 to 17.6 and from 6.4 to 10.3 , respectively, for O-620; from 5.3 to 8.3 and from 6.7 to 8.3 , respectively, for O-1369; and from 2.2 to 17.7 and from 6.4 to 10.3 , respectively, for O-2099.

Conclusions: DAT blockers have previously been shown to mitigate PD-like symptoms induced by MPTP in nonhuman primates. Here, we show that oral administration in mice of three DAT-selective blockers resulted in rapid absorption into the systemic circulation and the CNS. The CNS bioavailability and favorable brain-to-plasma ratios after oral dosing makes these DAT blockers attractive candidates for treatment of PD.

The work described here was conducted by QPS, L.L.C., for Alseres Pharmaceuticals. The compounds tested were synthesized for Alseres Pharmaceuticals by Organix, Inc.

\section{Poster Abstract \#13: Interactive Voice Response Diary and Objective Myotonia Measurement as Endpoints for Clinical Trials in Nondystrophic Interactive Voice Response Diary and Objective Myotonia Measurement as Endpoints for Clinical Trials in Nondystrophic Myotonia Richard Barohn*; CINCH Study Group ${ }^{\dagger}$ \\ *Department of Neurology, The University of Kansas Medical Center, Kansas City, $\mathrm{KS} ;{ }^{\dagger}$ Clinical Investigation of Neurological Channelopathies}

Background: Nondystrophic myotonia (NDM) is a heterogeneous group of neuromuscular disorders caused by mutations in skeletal muscle sodium and chloride channels. There are no established treatments for myotonia, despite the availability of agents that deserve careful study. An outcome measure of myotonia is important for clinical trials.

Methods: 45 subjects were enrolled from six academic centers across the United States, England, and Canada. Patients were categorized as myotonia congenita (MC), paramyotonia congenita (PMC), and other myotonic disorders (OMD) Three 
possible myotonia (symptoms/signs) measures were assessed: relaxation time after maximum voluntary isometric contraction of the finger flexors (QMA); myotonic discharges on needle EMG; an Interactive Voice Response Diary (IVR), for which participants called in once a week for up to 8 weeks to rank symptom severity on a scale of 1 to 9 (stiffness, pain, weakness, fatigue) and frequency (1-7 days).

Results: Initial clinical diagnosis was $20 \mathrm{MC}$; $16 \mathrm{PMC} ; 9$ OMD. Myotonic discharge potentials were seen in all subjects with no difference in their degree and location among the subtypes. Of 33 participants for whom QMA test results were available, 15 showed a delay in relaxation; however, for subjects with e 2's hand grip opening on clinical exam, 14/16 showed a delay in relaxation on QMA. From IVR data, frequency of symptoms for the total population was stiffness $91 \%$, pain $60 \%$, weakness $56 \%$, and fatigue $65 \%$. Average severity for each symptom was stiffness, 4.1; pain, 4.0; weakness, 4.2; and fatigue, 4.2. Standard deviation of reported symptom severity within subject was stiffness, 1.2; pain, 1.1; weakness, 1.3; and tiredness, 0.9. For participants reporting symptoms for a given week, the average number of days they experienced symptoms was stiffness, 5.2; pain, 5.2; weakness, 4.4; and fatigue, 4.5.

Conclusions: The IVR data showed consistency in patient self-rated clinical symptoms related to myotonia, which makes this a potentially useful endpoint for future clinical trials. Quantitive myotonia measure is a less sensitive outcome measure, because only minority patients showed abnormal results.

This project is supported by NIH grant 5U54RRO19482.

\section{Poster Abstract \#14: Magnetic Resonance Disease Severity Scale (MRDSS) Predicts Clinical Progression in Multiple Sclerosis}

Rohit Bakshi*; Mohit Neema, Brian C Healy,

Zsuzsanna Liptak, Rebecca A. Betensky, Guy J. Buckle,

Susan A. Gauthier, James Stankiewicz, Dominik Meier,

Svetlana Egorova, Ashish Arora, Zachary D. Guss,

Bonnie I. Glanz, Samia J. Khoury, Charles R.G. Guttmann, and Howard L. Weiner

*Departments of Neurology and Radiology, Brigham and Women's Hospital, Harvard Medical School, Boston MA

Objective: Combine MRI measures of disease severity into a composite score in patients with multiple sclerosis (MS).

Background: Brain MRI lesion and atrophy measures assessed individually have fairly weak ability to predict clinical progression in MS.

Methods: In the Comprehensive Longitudinal Investigation of MS at Brigham, we studied 103 patients [age (mean \pm SD) $42.7 \pm 9.1$ years, disease duration $14.1 \pm 9.2$ years, Expanded Disability Status Scale (EDSS) score $3.3 \pm 2.2,60 \%$ $(n=62)$ relapsing-remitting $(\mathrm{RR}), 32 \%(n=33)$ secondary progressive (SP), and $8 \%(n=8)$ primary progressive]. Brain MRI measures included baseline T2 hyperintense (T2LV) and T1 hypointense (T1LV) lesion volume, and brain parenchymal fraction (BPF), a marker of global atrophy. The ratio of T1LV to T2LV assessed lesion severity. A Magnetic Resonance Disease Severity Scale (MRDSS) score, on a continuous scale from 0 to 10 , was derived for each patient using T2LV, BPF, and $\mathrm{T} 1 / \mathrm{T} 2$ ratio.
Results: MRDSS score averaged $5.1 \pm 2.6$. Baseline MRI and EDSS correlations were moderate for BPF, T1/T2, and MRDSS and weak for T2LV. MRDSS showed a larger effect size than any of the individual MRI components in distinguishing RR from SP patients. Models containing either T2LV or MRDSS were significantly associated with EDSS disability progression during the $3.2 \pm 0.3$ year observation period, when adjusting for baseline EDSS score.

Conclusions: These results show that combining brain MRI lesion and atrophy measures can predict clinical progression in patients with MS and provide the basis to develop an MRI-based continuous scale as a marker of MS disease severity.

\section{Poster Abstract \#15: MRI Measurement of Whole Spinal Cord Volume at 3T: Reproducibility and Relevance to Multiple Sclerosis}

Ashish Arora, Mohit Neema, James Stankiewicz, Jason G Guss, and Rohit Bakshi

Brigham and Women's Hospital, Harvard Medical School, Boston MA

Objective: Determine the reproducibility and clinical relevance of measuring whole spinal cord volume in healthy controls and patients with multiple sclerosis (MS) using 3T MRI.

Background: Spinal cord atrophy may be especially relevant to disability in MS. The advent of 3T MRI brings the potential to assess spinal cord volume more precisely than conventional platforms.

Design/Methods: Eight patients with MS and five healthy subjects underwent 3T MRI of the whole spinal cord using axial fast spin-echo T2-weighted images. The upper cervical cord volume was calculated at $\mathrm{C} 2-3$. The cord volume was acquired using an edge-finding tool based on local thresholding and was normalized by the craniocaudal extent of the cord.

Results: The whole spinal cord volume in the MS group was $8.2 \%$ lower $v s$. healthy subjects $(p=0.13$; effect size, Cohen's $d=0.89$ ). Whole spinal cord volume normalized by number of slices was $5 \%$ lower in patients with MS $(p=0.16$; $d=0.79$ ) and whole spinal cord volume normalized by intracranial volume (ICV) was lower by $4.7 \%$ in MS $v s$. controls ( $p$ $=0.16 ; d=0.63$ ). Normalizing the whole cord volume with number of slices and ICV showed a $1.5 \%$ difference in MS vs. controls $(p=0.79 ; d=0.50)$. Upper cervical spinal cord volume was $7 \%$ lower in MS $v s$. controls $(p=0.33 ; d=0.51)$. The whole cord volume scan-rescan, intrarater, and inter-rater coefficient of variation ranged from $0.5 \%$ to $0.7 \%$; those for upper cervical cord cross-sectional volume ranged from $1.3 \%$ to $3.8 \%$.

Conclusions: In this pilot study, whole spinal cord volume shows larger effect sizes than upper cervical cord volume in differentiating mildly disabled patients with MS from normal controls. Normalization of spinal cord volume by intracranial volume reduced such effect sizes and therefore may not be desirable. Whole spinal cord volume showed higher reproducibility than measurement of upper cervical cord volume.

Study supported by: National Multiple Sclerosis Society (RG3705A1; RG3798A2); National Institutes of Health (NINDS 1R01NS055083-01). 